\title{
Nucleation in Sheared Granular Matter
}

\author{
Frank Rietz, ${ }^{1,2,3,4, *}$ Charles Radin, ${ }^{5}$ Harry L. Swinney, ${ }^{6}$ and Matthias Schröter ${ }^{1,2, \dagger}$ \\ ${ }^{1}$ Max-Planck-Institute for Dynamics and Self-Organization Göttingen, 37077 Göttingen, Germany \\ ${ }^{2}$ Institute for Multiscale Simulation, Friedrich-Alexander-Universität Erlangen-Nürnberg (FAU), 91052 Erlangen, Germany \\ ${ }^{3}$ Department of Nonlinear Phenomena, University Magdeburg, Universitätsplatz, 2, 39106 Magdeburg, Germany \\ ${ }^{4}$ Department of Pattern Formation, University Magdeburg, Universitätsplatz 2, 39106 Magdeburg, Germany \\ ${ }^{5}$ Department of Mathematics, University of Texas at Austin, Austin, Texas 78712, USA \\ ${ }^{6}$ Center for Nonlinear Dynamics and Department of Physics, University of Texas at Austin, Austin, Texas 78712, USA
}

(Received 9 June 2017; revised manuscript received 11 December 2017; published 2 February 2018)

\begin{abstract}
We present an experiment on crystallization of packings of macroscopic granular spheres. This system is often considered to be a model for thermally driven atomic or colloidal systems. Cyclically shearing a packing of frictional spheres, we observe a first order phase transition from a disordered to an ordered state. The ordered state consists of crystallites of mixed fcc and hcp symmetry that coexist with the amorphous bulk. The transition, initiated by homogeneous nucleation, overcomes a barrier at $64.5 \%$ volume fraction. Nucleation consists predominantly of the dissolving of small nuclei and the growth of nuclei that have reached a critical size of about ten spheres.
\end{abstract}

DOI: 10.1103/PhysRevLett.120.055701

Packings of spheres show interesting features such as phase transitions between disordered and ordered states, and can be useful in the study of amorphous atomic configurations [1]. Examples include thermal colloidal packings [2-4], packings of macroscopic granular spheres $[1,5-15]$, and simulations of the mathematical hard sphere model [16-25]. The behavior of such systems is determined by the fraction of space filled by the spheres, their packing fraction $\phi$.

The hard sphere model exhibits an entropically driven first order phase transition. Disordered fluid states are observed below the freezing density of $\phi=0.495$ and crystalline ordered states appear above the melting density of $\phi=0.545$, with coexistence of the two phases for intermediate densities [21].

Granular spheres can also be packed in disordered and ordered states. In contrast to colloidal packings and the mathematical hard sphere model, granular packings are characterized by the existence of permanent contacts between the particles. Most experimental protocols for increasing the bulk volume fraction, such as vertical shaking [12], centrifugation [4], thermal cycling [11] and sedimentation [13], do not achieve an ordered state from an initial disordered state. The "random close packed state" (rcp) is used operationally to describe the highest density state achieved by these methods. The rcp volume fraction is

Published by the American Physical Society under the terms of the Creative Commons Attribution 4.0 International license. Further distribution of this work must maintain attribution to the author(s) and the published article's title, journal citation, and DOI. in the range $0.635<\phi<0.655$ [14-17], about $15 \%$ lower than the densest possible packing of ordered face centered cubic (fcc) or hexagonal close packing (hcp), which each have a volume fraction $\phi=\pi / \sqrt{18} \approx 0.74$ [26].

Ordered clusters of granular spheres have been obtained with a system density $\phi_{\text {global }}$ in the range $0.64-0.74$ by multidimensional shaking $[5,6]$, cyclic shear $[7,8]$, and shear in a Couette cell [9]. By analogy with the freezingmelting transition in the hard sphere model, the emergence of growing crystallites found in our granular experiment can be interpreted in terms of nucleation and a first order phase transition $[20,27]$.

In our experiment we compact a granular packing by shearing. With increasing shear cycles, a well-defined plateau emerges at phase transition density $\phi_{\text {global }}=0.645$, which is in the range of densities associated with rcp [14-17]. Such a plateau was not reported in a previous experiment that used a setup similar to ours, and the growth rate of nuclei that we find differs qualitatively from that found in the previous experiment [8].

Our experiment uses a cubical shear cell with side length $10.5 \mathrm{~cm}$ [Fig. 1(a)]. Nucleation from the side walls is suppressed using half-spheres glued to the walls at random positions (glue: Vitralit 7562, Panacol). The cell is filled with 49400 precision BK7 glass spheres of diameter $3 \pm 0.0025 \mathrm{~mm}$ and density $2.51 \mathrm{~g} / \mathrm{cm}^{3}$ (size tolerance given by manufacturer, Worf Glaskugeln $\mathrm{GmbH}$ ). The top plate applies a pressure of $2.1 \mathrm{kPa}$ on the bed, and the pressure increases downward to $3.1 \mathrm{kPa}$ at the bottom. The cell is sheared by sinusoidally tilting opposite vertical shear walls by $\pm 0.6^{\circ}$ about axes indicated by red circles about halfway up opposite side walls [Fig. 1(a)]; the total 


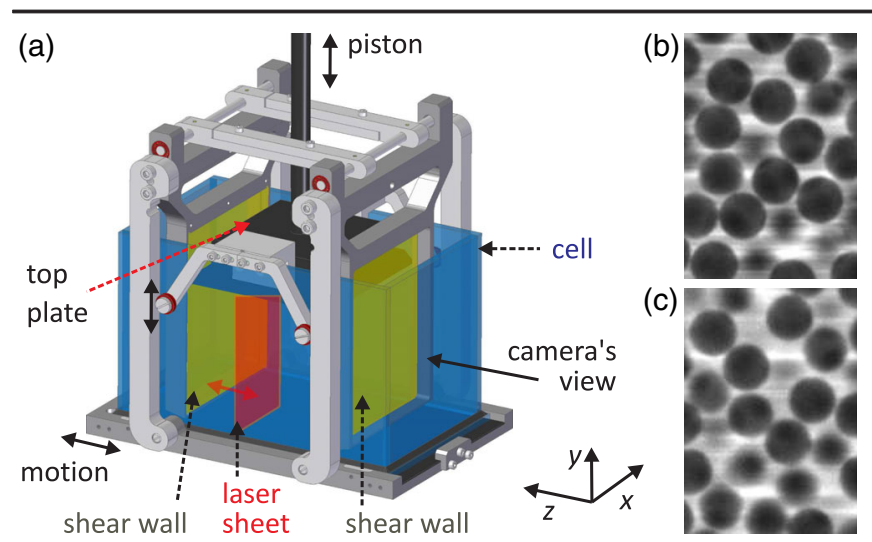

FIG. 1. Precision spheres are contained in a cubical volume with opposite side walls (lime green) that produce oscillating shear. The cell is filled with a liquid that is index matched to the beads. The top plate is levered to the side walls and is mounted on a piston that is constrained to move vertically. Shear is produced by periodically oscillating the cell bottom in the $z$ direction with a period of $2 \mathrm{~s}$. A laser sheet illuminates a slice in the $x-y$ plane, as illustrated by contrast enhanced images in (b) and (c); (b) shows a layer near the camera, and (c) shows a layer much further from the camera. Note that the image quality in both layers is comparable.

peak-to-peak oscillation displacement at the bottom of the cell is about one-third of a sphere diameter.

The glass spheres are index matched [28] to a mixture of phthalate esters (Cargille Laboratories, identifier code $1160, \rho=1.1 \mathrm{~g} / \mathrm{cm}^{3}, \nu=41 \mathrm{cSt}$ ) with a dissolved fluorescent dye (oxazine 750 perchlorate, $c=10 \mathrm{mg} / \mathrm{l}$ ). Hydrodynamic interactions are negligible for our small amplitude shear with a period of $2 \mathrm{~s}$, and sphere deformation is also negligible. The spheres, half-spheres, and shear cell walls are made of BK7 optical glass and are index matched with the liquid mixture in a temperature controlled environment $(n=1.5198 \pm 0.0001$ at $\lambda=589 \mathrm{~nm}$ and $\left.T=22.9 \pm 0.1^{\circ} \mathrm{C}\right)$.

The shearing process is periodically stopped to measure the packing using a horizontally translated vertical laser light sheet $(\lambda=658 \mathrm{~nm}, P=75 \mathrm{~mW})$. Fluorescent light is imaged simultaneously by the camera [Figs. 1(b) and 1(c)]. The image slices are combined to form a three-dimensional volume. The measurements presented here are from 618 scans made during a run with $1.955 \times 10^{6}$ cycles. In the course of the two month long run $10 \%$ of the spheres escaped from the sample cell through a gap between one of the shear walls and a side wall, and this possibly increased the mobility of spheres farther inside the packing, thereby enhancing their crystallization.

Positions of the 20000 spheres at least 3 diam from any wall are detected by convolution with a template. Then the peak of the correlation map in the $(x, y, z)$ directions is determined with $(37,37,30) \mathrm{pixel} /$ diam resolution using a three-point Gauss estimator [29]. This yields the position of each particle. From the pair correlation function we can
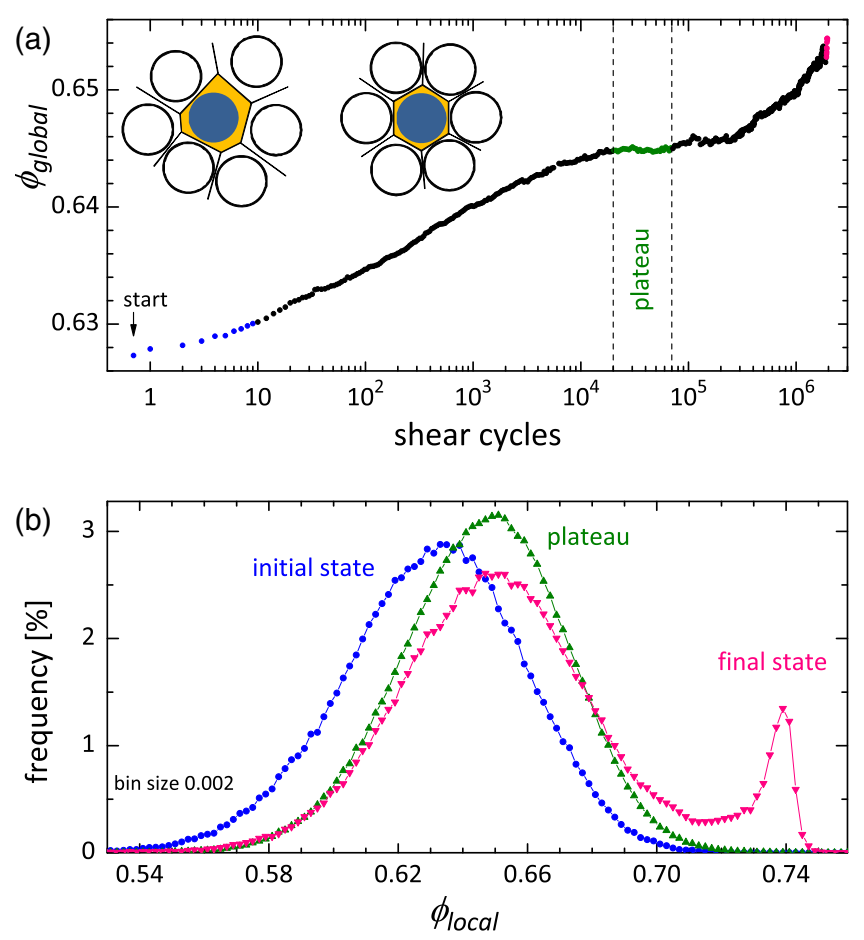

FIG. 2. The formation of the crystalline phase shows features of a first order phase transition. (a) Starting from a loose packing, the global volume fraction $\phi_{\text {global }}$ increases logarithmically with the number of shear cycles until it reaches a plateau at the density $\phi_{\text {global }}=0.645$. After approximately 50000 more shear cycles, $\phi_{\text {global }}$ increases again due to the formation of crystalline regions inside the sample. The 2D diagrams on the upper left show spheres (blue) and their Voronoi cells (yellow) for cases with Voronoi neighbors that are disordered loosely packed (left) and symmetric densely packed (right). (b) Histograms of local volume fractions reveal the coexistence of crystalline and amorphous regions inside the sample. Below the transition there is only a single peaked distribution, which shifts towards higher densities until the plateau is reached. After the onset of crystallization the previous peak population diminishes and a new peak appears at $\phi_{\text {local }}=0.74$. The histogram for the plateau is the average of 32 consecutive scans; elsewhere the histogram is the average of 10 scans. Colors in (b) correspond to the colored data points in (a).

determine, for more than $99 \%$ of the particles, the position of each particle to less than $2 \%$ of a sphere diameter (see Supplemental Material [30]).

For each sphere there is a Voronoi cell consisting of all points closer to that sphere than to any other sphere in the sample [see insets in Fig. 2(a)]. $\phi_{\text {local }}$ is then the ratio of the sphere volume to the volume of its Voronoi cell. The mean volume fraction of the whole sample, $\phi_{\text {global }}$, is given by the harmonic mean of all $\phi_{\text {local }}$ values [34].

The angular order between spheres sharing a face of their Voronoi cells is characterized by a weighted version of the order parameter $q_{6}$ [19]. A sphere is called crystalline if it is densely packed and its neighbors are ordered, i.e., $\phi_{\text {local }}>$ 0.72 and $q_{6}$ is either in the range $q_{6}(\mathrm{fcc})=0.575 \pm 0.020$ 

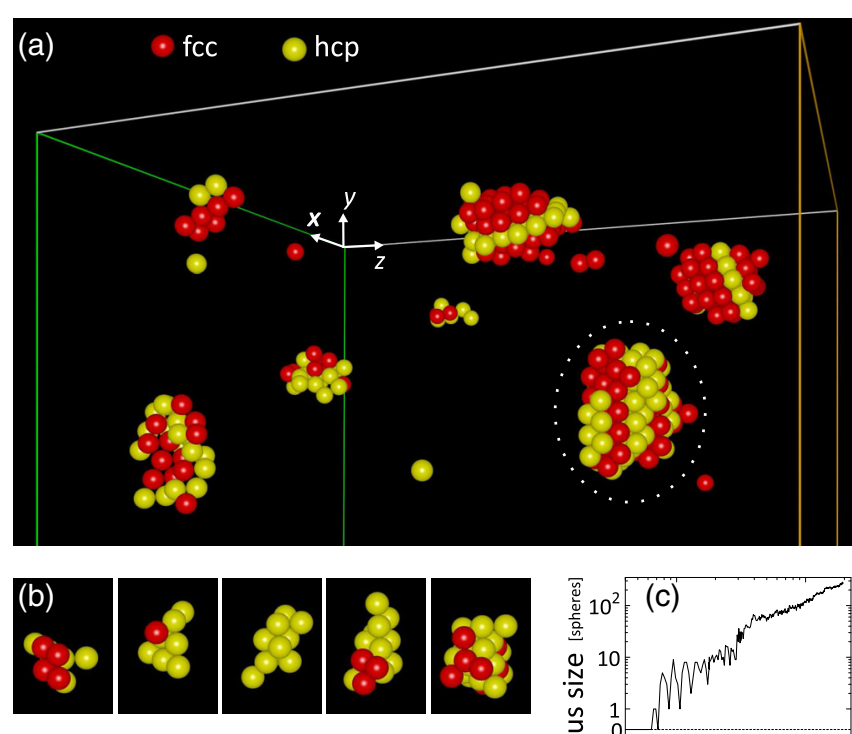

spheres $9 \quad 8$

cycles $94 \mathrm{k} \quad 144 \mathrm{k}$

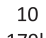

$179 \mathrm{k}$

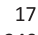

$240 \mathrm{k}$

29

$314 \mathrm{k}$

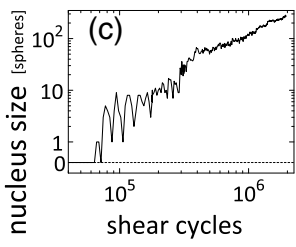

FIG. 3. (a) This cross section of the cell after $10^{6}$ cycles shows crystalline regions in the cell interior; the amorphous phase is not shown. The colors of the nucleated spheres indicate crystal type. The wire frame indicates the inner part of the shear cell that is used for evaluation. (b) The nucleus that started to grow first [dotted ellipse in (a)] fluctuated in shape and size until a stable seed was reached at about $3 \times 10^{5}$ cycles. (c) Time evolution of the nucleus in (b). A movie in the Supplemental Material [30] illustrates the nucleation in the shear cell.

or $q_{6}(\mathrm{hcp})=0.485 \pm 0.020$. Other spheres are called amorphous. A nucleus is a connected set of crystalline spheres, each sharing at least one Voronoi face with another sphere in the set. Some authors suggest different definitions for local order [16,35], but our results do not depend qualitatively on the choice of definition or threshold (see Supplemental Material [30] and a recent review [36]). The distance of a nucleus to another single sphere or nucleus is given by the shortest distance between the sphere centers.

During densification we observe three distinct regions, as can be seen in Fig. 2(a). The packing starts from a disordered state and compacts approximately logarithmically with time for about 20000 cycles. The compaction then slows to a stop, and the second region, a plateau $\left(\phi_{\text {global }}=0.645\right)$, emerges and persists for about 50000 cycles. Then the first growing nucleus appears, indicating a first order phase transition to the third distinct region. The volume fraction slowly begins to increase as shearing continues, and nuclei increase in number and size but have no preferred orientation of their hexagonal layers; an intermediate state of the system is shown in Fig. 3(a). The first growing nucleus is shown in Fig. 3(b), which illustrates that nuclei fluctuate in shape, size, and crystal symmetry as they grow, as can be seen in the movie in the Supplemental Material [30].
All nuclei start their growth at least 10 sphere diam distance away from any wall. By the end of the experiment, after $2 \times 10^{6}$ shear cycles, nuclei with up to $\sim 600$ spheres are present, and $9 \%$ of all spheres in the analyzed volume are in crystallites, which have fcc or hcp symmetry with approximately equal probability; no icosahedral symmetry was observed (see Supplemental Material [30]).

Histograms of local densities for amorphous packings have a single peak and are approximately symmetrical about that peak, as Fig. 2(b) illustrates. During compaction the peak narrows slightly and shifts to higher densities. Beyond the first order phase transition a second peak emerges at $\phi_{\text {local }}=0.74$, the density of densest packed arrangements.

The end of the densification plateau is identified by the emergence of a nucleus in the interior region; subsequently, all nuclei are tracked for each successive scan. Nuclei with fewer than about ten spheres are found to shrink more often than grow, while growth gradually becomes more probable for nuclei with more than ten spheres, that is, $\Delta \rho=\left(\rho_{\text {grow }}-\rho_{\text {shrink }}\right)>0$, where $\rho$ is the probability to grow or shrink, respectively [3,8] [Fig. 4(a)]. The critical size at which the difference of the probabilities $\Delta \rho$ becomes positive does not depend on the definition of local crystallinity (see Supplemental Material [30]).

Our main result is the observation of nucleation centers throughout the interior of a granular packing. The emergence of a plateau at a well-defined packing fraction, $\phi_{\text {global }}=0.645$, indicates the onset of a first order phase transition. A previous experiment used a setup similar to ours and observed heterogenous nucleation at the cell walls but no nucleation in the interior of the cell [8]. Another experiment used shaking in a spherical container which may or may not have suppressed heterogeneous nucleation; however, the spatiotemporal evolution of the nuclei was not determined in this study (see Refs. $[5,6]$ and Supplemental Material in Ref. [5]).

Our experiment analyzes static granular matter between episodes of cyclic shearing, which increase the density. Our data are snapshots, measurements of static packings. When the snapshots are viewed consecutively, as in a movie, nuclei emerge, grow, and shrink until after sufficient shearing cycles, nuclei of critical size are created that then grow indefinitely under further shearing (see movie in Supplemental Material [30]). A nucleation theory should explain why shearing at low amplitude and low frequency, in the presence of pressure and gravity, leads to such a sequence of static granular configurations. This is distinct from the usual nucleation theory, which shows that a thermal or Brownian dynamics, starting from a supercooled state, leads to the creation, growth, and shrinking of nuclei.

The observed compaction of a granular bed under gravity and pressure can be described by a simple mechanical picture. A bed of frictional hard spheres increases in density if subjected to repeated small disturbances that 

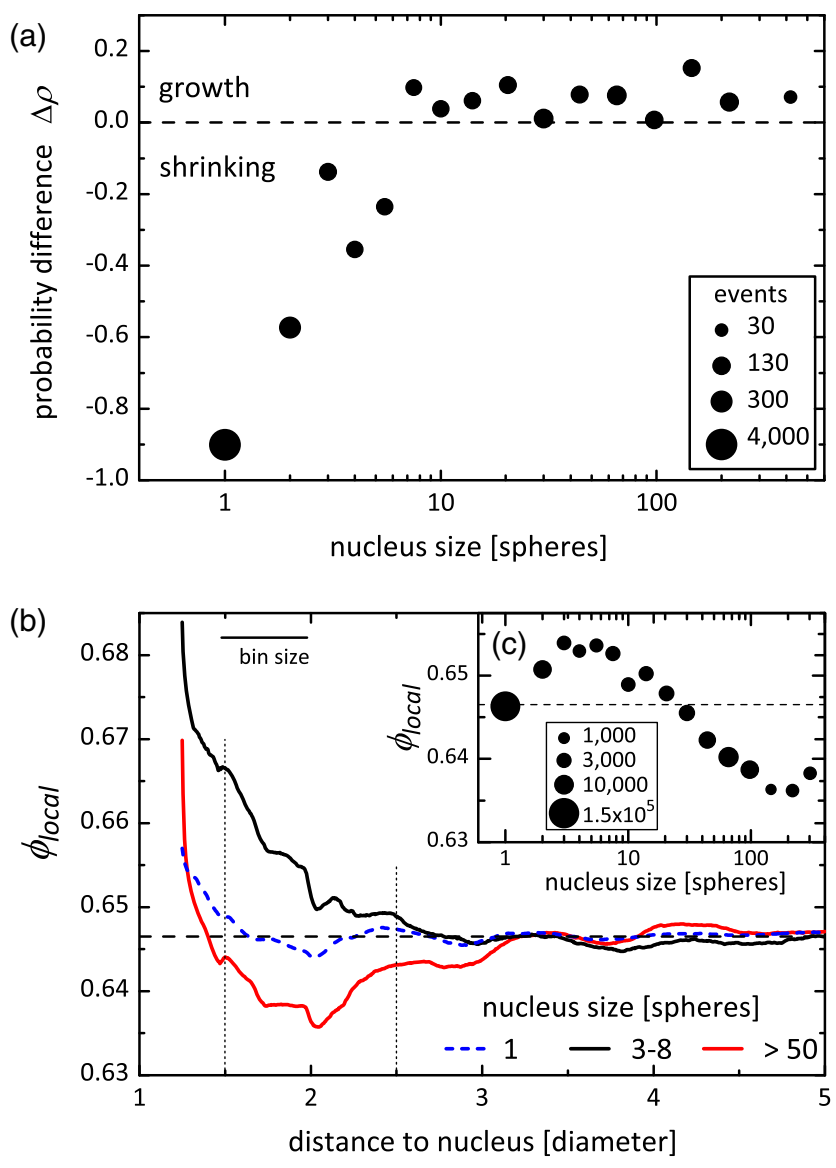

FIG. 4. (a) The growth (shrinking) probability of a nucleus depends on its size: nuclei with about ten or more spheres predominately grow $(\Delta \rho>0)$. (b) Local volume fraction as a function of distance from the surface of a nucleus. Large nuclei (red) show a minimum at a distance of about 2 sphere diam, but there is no comparable minimum for subcritical nuclei (black), and very small nuclei have a minor effect on the surroundings (dashed). (c) The packing density near the surface of a nucleusin the range in (b) between the vertical dotted lines - is higher for the small nuclei that tend to dissolve than for the large nuclei that tend to grow (see Figs. S3-S12 in the Supplemental Material [30]). The horizontal dashed line in (b) and (c) indicates the amorphous plateau far from any nucleus.

are introduced, for example, by shearing, shaking, or fluidization. The repeated shear cycles in our experiment break some of the force chains that form a skeleton supporting the bed, and this leads to compaction [24]. However, this simple picture does not address the homogeneous nucleation seen in our experiment [Fig. 4(a)]; very dense crystallite clusters $(\phi=0.74)$ form, grow, and dissolve without the help of a flat wall. Figure 4(b) shows that near a small nucleus the volume fraction is slightly higher than the background volume fraction, which suggests from a mechanical argument that under a confining pressure small nuclei should grow. However, Fig. 4(a) reveals that this does not happen. Any theory for nucleation in our system would have to account for these results in Fig. 4.
Our results are qualitatively different from the crystallization dynamics observed in constant volume molecular dynamics simulations of supercooled hard spheres: the existence of a nucleation barrier in our system disagrees with the autocatalytic growth of crystalline regions observed in Ref. [23]. Further, we do not observe burstlike growth events as seen in mature glasses [24,25]. These differences indicate that nucleation in our sheared granular system is governed by its own dynamics.

In conclusion, we have found that small amplitude cyclic shear of a bed of spherical particles under gravity and pressure leads to compaction until a well-defined randomclose-packed volume fraction is reached. Then after many more cycles (50 000 in our experiment), clusters with hcp and fcc symmetry emerge, and these crystallites grow if they contain about ten or more particles. Previous experiments [5-9] had observed nucleation centers on flat confining walls, but our experiment shows nucleation throughout the interior of the cell. Further, the present experiment on 49400 spheres indicates that future numerical simulations of sheared packings should extend well beyond a recent study of 2000 frictional grains cyclically sheared for 2000 cycles [18].

We thank Markus Benderoth, Thomas Eggers, Tilo Finger, Kristian Hantke, Wolf Keiderling, Udo Krafft, and Vishnu Natchu for technical support and discussions. The project was financed by grants from the German Academic Exchange Service (DAAD), Deutsche Forschungsgemeinschaft (DFG) through the Cluster of Excellence Engineering of Advanced Materials, and in part by NSF DMS-1208941, NSF DMS-1509088, and Robert A. Welch Foundation Grant F-0805.

*frank.rietz@gmx.net †matthias.schroeter@ds.mpg.de

[1] J. L. Finney, Philos. Mag. 93, 3940 (2013).

[2] P. N. Pusey and W. v. Megen, Nature (London) 320, 340 (1986).

[3] U. Gasser, E. R. Weeks, A. Schofield, P. N. Pusey, and D. A. Weitz, Science 292, 258 (2001).

[4] S. R. Liber, S. Borohovich, A. V. Butenko, A. B. Schofield, and E. Sloutskin, Proc. Natl. Acad. Sci. U.S.A. 110, 5769 (2013).

[5] N. Francois, M. Saadatfar, R. Cruikshank, and A. Sheppard, Phys. Rev. Lett. 111, 148001 (2013).

[6] M. Hanifpour, N. Francois, S. M. Vaez Allaei, T. Senden, and M. Saadatfar, Phys. Rev. Lett. 113, 148001 (2014); M. Hanifpour, N. Francois, V. Robins, A. Kingston, S. M. Vaez Allaei, and M. Saadatfar, Phys. Rev. E 91, 062202 (2015); M. Saadatfar, H. Takeuchi, V. Robins, N. Francois, and Y. Hiraoka, Nat. Commun. 8, 15082 (2017).

[7] D. Bernal, K. R. Knight, and I. Cherry, Nature (London) 202, 852 (1964); M. Nicolas, P. Duru, and O. Pouliquen, Eur. Phys. J. E 3, 309 (2000); N. W. Mueggenburg, Phys. Rev. E 71, 031301 (2005). 
[8] A. Panaitescu, K. A. Reddy, and A. Kudrolli, Phys. Rev. Lett. 108, 108001 (2012).

[9] J.-C. Tsai, G. A. Voth, and J. P. Gollub, Phys. Rev. Lett. 91, 064301 (2003); K. E. Daniels and R. P. Behringer, J. Stat. Mech. (2006) P07018.

[10] Y. Komatsu and H. Tanaka, Phys. Rev. X 5, 031025 (2015).

[11] K. Chen, J. Cole, C. Conger, J. Draskovic, M. Lohr, K. Klein, T. Scheidemantel, and P. Schiffer, Nature (London) 442, 257 (2006); S. Slotterback, M. Toiya, L. Goff, J. F. Douglas, and W. Losert, Phys. Rev. Lett. 101, 258001 (2008).

[12] J. B. Knight, C. G. Fandrich, C. N. Lau, H. M. Jaeger, and S. R. Nagel, Phys. Rev. E 51, 3957 (1995); P. Richard, M. Nicodemi, R. Delannay, P. Ribiere, and D. Bideau, Nat. Mater. 4, 121 (2005).

[13] M. Schröter, D. I. Goldman, and H. L. Swinney, Phys. Rev. E 71, 030301(R) (2005).

[14] G. D. Scott, Nature (London) 188, 908 (1960); G. D. Scott and D. M. Kilgour, J. Phys. D 2, 863 (1969); J. L. Finney, Proc. R. Soc. A 319, 479 (1970).

[15] J. G. Berryman, Phys. Rev. A 27, 1053 (1983).

[16] S. C. Kapfer, W. Mickel, K. Mecke, and G. E. SchröderTurk, Phys. Rev. E 85, 030301(R) (2012).

[17] A. V. Anikeenko and N. N. Medvedev, Phys. Rev. Lett. 98, 235504 (2007); V. Baranau and U. Tallarek, Soft Matter 10, 3826 (2014).

[18] J. R. Royer and P. M. Chaikin, Proc. Natl. Acad. Sci. U.S.A. 112, 49 (2015).

[19] W. Mickel, S. C. Kapfer, G. E. Schröder-Turk, and K. Mecke, J. Chem. Phys. 138, 044501 (2013).

[20] Y. Jin and H. A. Makse, Physica (Amsterdam) 389A, 5362 (2010).

[21] W. W. Wood and J. D. Jacobson, J. Chem. Phys. 27, 1207 (1957); B. J. Alder and T. E. Wainwright, J. Chem. Phys. 27, 1208 (1957); W. G. Hoover and F. H. Ree, J. Chem. Phys. 49, 3609 (1968).

[22] S. Auer and D. Frenkel, J. Chem. Phys. 120, 3015 (2004).
[23] E. Sanz, C. Valeriani, E. Zaccarelli, W. C. K. Poon, P. N. Pusey, and M.E. Cates, Phys. Rev. Lett. 106, 215701 (2011); C. Valeriani, E. Sanz, P. N. Pusey, W. C. K. Poon, M. E. Cates, and E. Zaccarelli, Soft Matter 8, 4960 (2012).

[24] T. Yanagishima, J. Russo, and H. Tanaka, Nat. Commun. 8, 15954 (2017).

[25] E. Sanz, C. Valeriani, E. Zaccarelli, W. C. K. Poon, M. E. Cates, and P. N. Pusey, Proc. Natl. Acad. Sci. U.S.A. 111, 75 (2014).

[26] T. C. Hales, Dense Sphere Packings. A Blueprint for Formal Proofs (Cambridge University Press, Cambridge, England, 2012).

[27] C. Radin, J. Stat. Phys. 131, 567 (2008); D. Aristoff and C. Radin, J. Math. Phys. (N.Y.) 51, 113302 (2010).

[28] J. A. Dijksman, F. Rietz, K. A. Lőrincz, M. v. Hecke, and W. Losert, Rev. Sci. Instrum. 83, 011301 (2012).

[29] M. Raffel, C. E. Willert, S. T. Wereley, and J. Kompenhans, Particle Image Velocimetry (Springer, Berlin, 2007), p. 160.

[30] See Supplemental Material at http://link.aps.org/ supplemental/10.1103/PhysRevLett.120.055701 for a movie and additional evaluations, which includes Refs. [31-33].

[31] P. J. Steinhardt, D. R. Nelson, and M. Ronchetti, Phys. Rev. B 28, 784 (1983).

[32] B. A. Klumov, Y. Jin, and H. A. Makse, J. Phys. Chem. B 118, 10761 (2014).

[33] M. Leocmach and H. Tanaka, Nat. Commun. 3, 974 (2012).

[34] S. Weis and M. Schröter, Rev. Sci. Instrum. 88, 051809 (2017).

[35] D. Faken and H. Jónsson, Comput. Mater. Sci. 2, 279 (1994); M. Bargieł and E. M. Tory, Adv. Powder Technol. 12, 533 (2001); A. Stukowski, Model. Simul. Mater. Sci. Eng. 20, 045021 (2012); H. Tanaka, Eur. Phys. J. E 35, 113 (2012); M. Leocmach, J. Russo, and H. Tanaka, J. Chem. Phys. 138, 12 A536 (2013).

[36] W. F. Reinhart, A. W. Long, M. P. Howard, A. L. Ferguson, and A. Z. Panagiotopoulos, Soft Matter 13, 4733 (2017). 